\title{
From the Editor-in-Chief Reza Shaker
}

Dear Dysphagia Friends,

I am writing to report that starting with the current issue, Dysphagia will publish bi-monthly instead of quarterly. This is a significant milestone in the history of the Journal. Thanks to your support Dysphagia has experienced a steady rise in the number of submitted papers over the past several years which necessitated the increase in the number of issues to accommodate the number of papers that achieve acceptance through a rigorous review process.

We are indebted to our dedicated Associate Editors, Editorial Board members and our Editorial, Production and Publication staff for their tireless work to maintain the review and publication processes on track.

Since its inception, Dysphagia has served as a multidisciplinary platform for all those who wish to contribute to the art and science of Deglutology and deglutition disorders. It has played an invaluable role in dissemination of knowledge in physiology and pathophysiology of swallowing, new diagnostic and therapeutic modalities, dysphagia related public health issues and more importantly in the emergence of the trans-disciplinary field of Deglutology addressing the entire phases of swallowing.
Deglutology, like no other field, is in need of convergence of various contributory disciplines to develop a trans-disciplinary model to further improve patient care, advance research and facilitate education. As a multidisciplinary international journal, Dysphagia has facilitated this process and has made it possible for the clinicians and researchers to gain access to state-of-the-art knowledge from different disciplines and as such develop a truly multidisciplinary perspective on swallowing and its disorders. Dysphagia has given voice to dysphagic patients and recognition to dysphagia researchers and clinicians worldwide.

It is anticipated that, with bi-monthly publication, Dysphagia will contribute further to the art and science of Deglutology and will enhance its service to the dysphagia patients, and deglutologists alike.

Best regards,

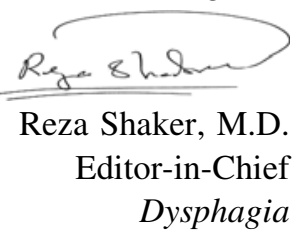

